\title{
Effects of Statins on Lipid Profile of Kidney Transplant Recipients: A Meta-Analysis of Randomized Controlled Trials
}

\author{
Xiu Huang $\mathbb{D},{ }^{1}$ Yong Jia, ${ }^{2}$ Xiaoyu Zhu ${ }^{\mathbb{D}},{ }^{1}$ Yangyang Zhang, ${ }^{1}$ Lili Jiang, ${ }^{1}$ Xuejiao Wei, ${ }^{1}$ \\ Dan Zhao, ${ }^{1}$ Xiaoxia Zhao, ${ }^{1}$ and Yujun Du $\mathbb{D}^{1}$ \\ ${ }^{1}$ Department of Nephrology, The First Hospital of Jilin University, Changchun 130000, China \\ ${ }^{2}$ School of Nursing, Jilin University, Changchun 130000, China \\ Correspondence should be addressed to Yujun Du; kjkduyujun@126.com
}

Received 15 December 2019; Revised 21 March 2020; Accepted 7 April 2020; Published 2 May 2020

Academic Editor: José L. Campos

Copyright (C) 2020 Xiu Huang et al. This is an open access article distributed under the Creative Commons Attribution License, which permits unrestricted use, distribution, and reproduction in any medium, provided the original work is properly cited.

\begin{abstract}
Objective. To assess the benefits of statins on lipid profile in kidney transplant recipients via a meta-analysis. Methods. We systematically identified peer-reviewed clinical trials, review articles, and treatment guidelines from PubMed, Embase, the Cochrane Library, Wanfang, Chinese National Knowledge Infrastructure (CNKI), SinoMed (CBM), and Chongqing VIP databases from inception to April 2019. In the analysis, only randomized controlled clinical trials performed in human were included. Results. Eight articles were included in the analysis, involving 335 kidney transplant recipients who received statins and 350 kidney transplant patients as the control group. Results revealed that statins improved the lipid profile of kidney transplant recipients. Specifically, statin therapy significantly reduced total cholesterol and low-density lipoprotein cholesterol. However, it had no effects on high-density lipoprotein cholesterol and triglyceride levels. Conclusions. The present study provides valuable knowledge on the potential benefits of statins in kidney transplant recipients. This meta-analysis shows that statin therapy modifies the lipid profile in this patient population.
\end{abstract}

\section{Introduction}

The burden of chronic kidney disease (CKD) is rapidly increasing worldwide. Studies have reported that cardiovascular disease (CVD) is a leading cause of death in patients with CKD, especially kidney transplant recipients (KTRs) [1]. It has been estimated that the mortality rate of CVD is $17-50 \%$ in KTRs [2]. Previously, it was found that there are many risk factors contributing to CVD. Among the important risk factors for cardiovascular disease are high level of atherosclerosis, ageing, heightened systemic inflammation, oxidative stress, endothelial dysfunction, hypertension, insulin resistance and diabetes mellitus, cigarette smoking, and dyslipidemia. Data show that dyslipidemia is one of the most common metabolic complications and the incidence of it is as high as $80 \%$ in KTRs [3].

Dyslipidemia is not only associated with high CVD risk but is also an independent risk factor for allograft rejection and graft survival in KTRs [4]. Awareness of the harm of posttransplant dyslipidemia and optimization of suitable therapeutic strategies are crucial to KTRs. Therefore, it is reasonable to assume that the intervention of dyslipidemia could improve lipid profile in KTRs.

Statins (inhibitors of 3-hydroxy-3-methylglutaryl coenzyme A (HMG-CoA) reductase) may be a good choice for the primary and secondary prevention drugs of CVD and the treatment of lipid profile. There are a series of studies that have focused on lipid profile in all CKD; however, few studies are about KTRs that are an overlooked group and there are well discrepancies between the results of these studies. For example, Palmer et al. [5] showed that statins can significantly reduce serum total cholesterol (TC) and low-density lipoprotein cholesterol (LDL-C) and lower high-density lipoprotein cholesterol (HDL-C) and serum triglycerides (TG). However, Messow and Isles [6] held that lowering lipid through statins was not effective in patients after renal transplant.

The reasons that we do not replicate these studies are as follows: firstly, the benefits of statins on lipid profile in kidney 
transplant recipients remain controversial so far, and secondly, in the last few years, there have been some original studies about it that have been published. Lastly, we have already searched the Chinese databases that were different from other meta-analyses. Thus, this meta-analysis is aimed at critically appraising scientific evidence about the efficacy of statins on lipid profile in KTRs.

\section{Methods}

2.1. Eligibility Criteria. Only RCTs were inclusive within this review. Cross-sectional studies, cohorts, case studies, and crossover experiments were excluded to be able to determine the effectiveness of statin intervention. The study population was adults (more than 18 years) who have received only kidney transplant without multiorgan transplant for over 1 month. The intervention was statin therapy involving all kinds of statins. The control group comprised patients receiving placebo, routine care, and no treatment. The primary outcome was serum lipid level including TC, LDL-C, HDL-C, and TG.

2.2. Data Sources and Search Strategy. All publications were searched on English databases which included the PubMed, Cochrane Library, Embase, and the Chinese databases which included the Wanfang database, Chinese National Knowledge Infrastructure (CNKI), VIP database, and SinoMed from inception to April 2019 without language restriction.

The following search terms were used: renal transplantation, kidney transplantation, acute renal allograft rejection, renal allograft rejection, hypercholesterolemia, hyperlipidemia, dyslipidemia, hydroxymethylglutaryl coenzyme a reductase inhibitor, HMG-CoA reductase inhibitors, hydroxymethylglutaryl-CoA reductase inhibitors, statin, pravastatin, fluvastatin, lovastatin, simvastatin, atorvastatin, cerivastatin, and rosuvastatin. Further details of the search strategies are available from the authors by request. The reference lists of identified trials and review articles were scrutinized for additional trials. Draft Embase and SinoMed search strategies are included as Supplementary Material 1. All studies included in the meta-analysis were randomized controlled clinical trials.

2.3. Study Selection. Eligible studies were identified by screening the titles and abstracts. Two reviewers (Ms. Huang and Ms. Zhu) independently screened all databases to retrieve studies that met the eligibility criteria. Disagreements on whether an article was eligible were resolved by discussion or were arbitrated by a third reviewer (Mr. Jia).

2.4. Data Extraction. The following data were extracted from eligible trials: author's name, year of publication, types of statins, duration, the dose of statins, the number of total patients, the duration of follow-up, and the level of TC, LDL-C, HDL-C, and TG after treatment. The extraction results were reanalyzed by two independent reviewers. Discrepancies were solved by discussion or through reevaluation by a third reviewer.
2.5. Risk of Bias. The Cochrane Collaboration's tool was utilized to assess the bias risk in all the included studies. The following sources of bias were recorded: method of randomization, allocation concealment, blinding of participants and personnel, binding of outcome assessment, incomplete outcome data, selective reporting bias, and other bias. Each article was categorized either as "high risk," "low risk," or "unclear risk," with the last category meaning that either lack of information or unclear about the potential for bias.

2.6. Statistical Analysis. Included studies were weighted by effect size and pooled. The Review Manager software 5.3 was used to assess standardized mean differences (SMDs) with $95 \%$ confidence intervals (95\%CIs) which were calculated using DerSimonian and Laird (for random effects) methods. The Cochran $Q$ test and the $I^{2}$ statistic with $95 \%$ CIs were performed to determine the level of heterogeneity [7], and statistical significance was defined as $p<0.05$. Given that an $I^{2}$ equaling 0 does not exist, values of $95 \%$ CIs $\geq 50 \%$ were used to represent high heterogeneity [8]. The randomeffects model was applied in the meta-analysis. Funnel plots that were calculated in the Review Manager software were utilized to indicate publication bias. Subgroup analyses were not performed because of the limited number of studies.

\section{Results}

3.1. Study Selection. The search identified 7278 records of which 2030 potentially relevant full articles were found to be eligible (Figure 1). Finally, eight studies met the inclusion criteria [9-16], covering 350 patients who received kidney transplant and 335 patients randomized to the control group.

3.2. General Characteristics of the Studies Included in the Meta-Analysis. All studies were published between 1995 and 2015. The number of participants ranged from 20 to 229. The treatment duration was as follows: one study had 4-week treatment [12], one study had 12-week treatment [16], three studies had 3-week treatment duration $[10,13,17]$, and three studies had 6-week treatment duration $[9,14,15]$. All participants underwent kidney transplantation. In all studies, patients received statin therapy. The statins were prescribed at different clinical dosages ranging from 10 to $80 \mathrm{mg}$ including, fluvastatin, simvastatin, pravastatin, and atorvastatin. The follow-up duration was reported in all studies. The general characteristics of each included study are listed in Table 1.

3.3. Risk of Bias. The summary of risk of bias in each study is presented in Figure 2.

3.4. Outcome Analysis. The analysis identified high heterogeneity among the studies. Because the studies could not be combined, a random-effects model was used to pool the effect size for SMDs and 95\% CIs for the analysis.

3.4.1. The Effects of Statins on TC in KTRs. Eight trials reported the effect size of TC in KTRs (Table 1). The data show that the SMD was -2.90 with $95 \%$ CI of -4.52 to- 1.27 compared to the control group $(p=0.0005$, Figure 3$)$. The 


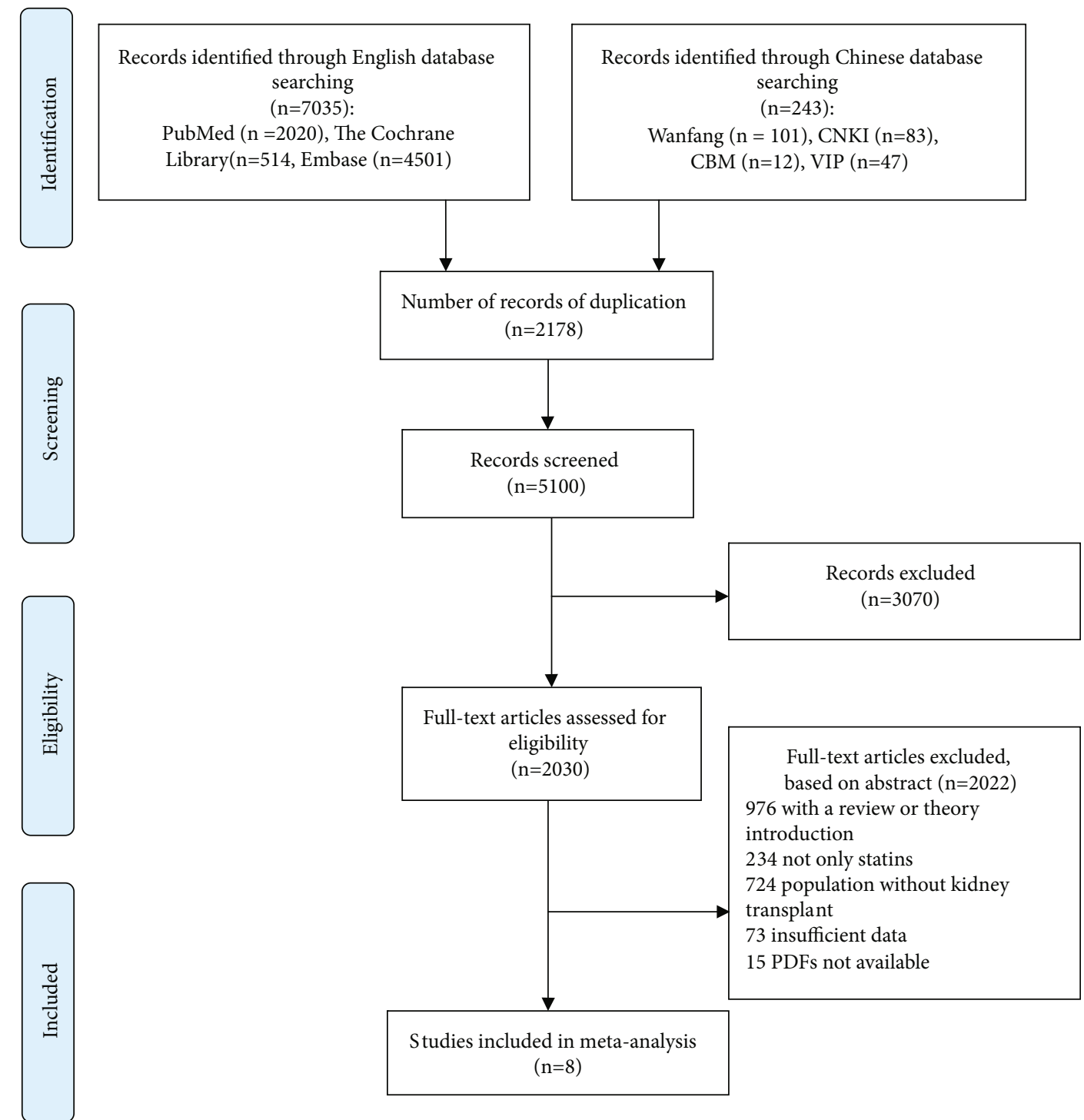

FIgURE 1: Flow chart of the selection process for eligible studies.

$I^{2}$ value was $98 \%$, suggesting the presence of heterogeneity. In addition, results revealed that statin therapy lowered TC levels.

3.4.2. The Effect of Statins on LDL-C in KTRs. Eight trials reported the effect size of LDL-C in KTRs who received statin therapy (Table 1). The analysis revealed that the SMD was -3.35 with $95 \%$ CI of -5.52 to -1.45 compared to the control group (Figure 4). Although the level of heterogeneity was high $\left(I^{2}=98 \% ; p<0.00001\right)$, the difference between the control group and the experimental group was statistically significant $(p=0.0005)$. The results showed that statin therapy decreased the level of LDL-C compared to the controls.

3.4.3. The Effects of Statins on HDL-C and TC in KTRs. Eight trials reported the level of HDL-C and TC (Table 1). The analysis revealed that there was no heterogeneity and none of them have statistical significance. The SMD of HDL-C was -0.23 with $95 \%$ CI of -1.27 to $0.82(p=0.67$, Figure 5$)$, and the SMD of TG was -0.39 with $95 \%$ CI of -1.45 to 0.66 $(p=0.47$, Figure 6). Results showed that statin therapy did not improve the profile of HDL-C or TG in KTRs.

3.5. Sensitivity Analysis. Each study was individually excluded to determine the impact of each study on the outcomes. Because of the high heterogeneity, sensitivity analysis was performed for TC and LDL-C. Exclusion of studies by Holdaas et al. [10] and Kasiske et al. [11] decreased the heterogeneity level from $98 \%$ to $91 \%$ for TC and from $98 \%$ to 93\% for LDL-C. The conclusions made were not changed when studies with high risk of bias were omitted.

\section{Discussion}

4.1. Basic Findings. This meta-analysis was performed on eight studies covering 350 kidney transplant patients who received statins and 335 kidney transplant patients as the control group. The aim of this study was to determine the 


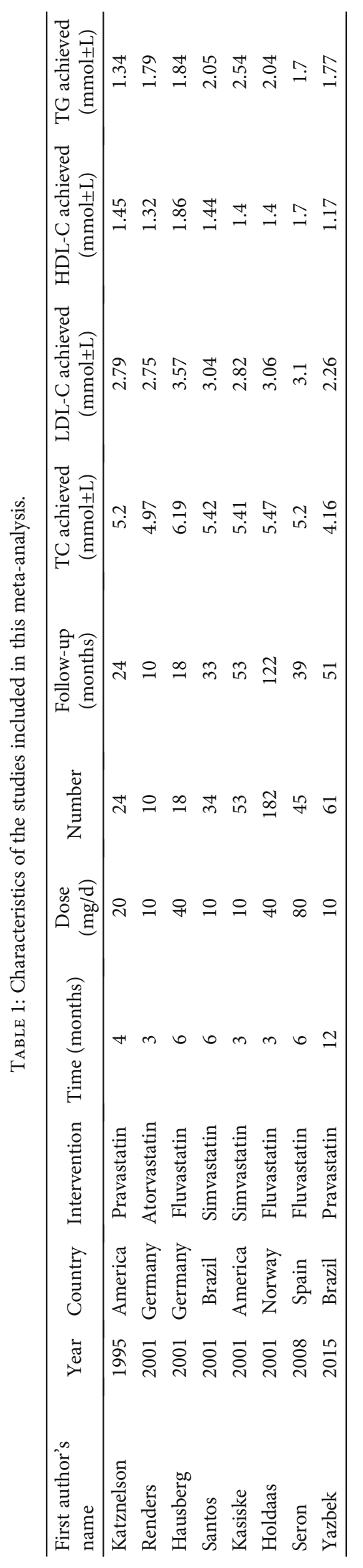




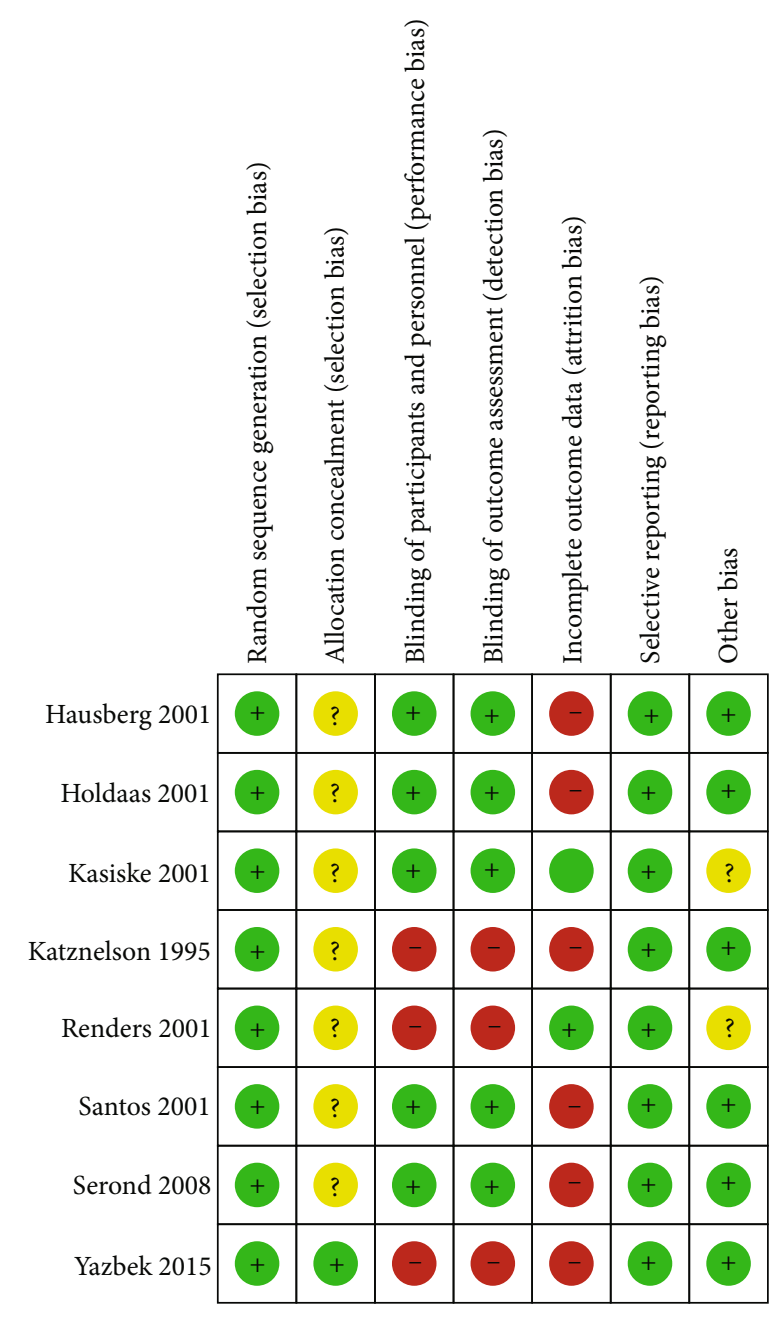

Figure 2: Summary of the risk of bias.

clinical value of statins on the lipid profile of KTRs. It was observed that statin therapy (including many types of statins) reduced serum levels of TC and LDL-C, which is consistent with previous reports [5]. However, statins had no significant effect on HDL-C and TG. Given the presence of risk of bias and the limited data, the findings of this analysis might not be conclusive. In addition, the effects of statins on cardiovascular events, cerebrovascular events, all-cause mortality, and the specific characteristics could be analyzed. Large-scale and high-quality trials are required to evaluate the impact of statins on these parameters in KTRs.

So far, some large-scale studies have reported the effects of statins on CKD. The German Diabetes and Dialysis Study (4D) [18] covering 1255 type 2 diabetes mellitus subjects who received maintenance hemodialysis found that the median level of LDL-C was reduced by $42 \%$ among patients receiving atorvastatin after four weeks of treatment. Another study evaluated the efficacy of rosuvastatin in 2776 patients undergoing regular hemodialysis (AURORA) [19]. They reported that administration of statin therapy for 3 months reduced the mean level of LDL-C by $43 \%$. However, few studies have determined the influence of statin therapy on KTRs. In the ALERT trial [20], 2012 KTRs were enrolled and followed up for about five to six years. It was noted that the use of statins improved cholesterol levels in patients and increased the survival rate by $24 \%$ compared to patients who did not receive fluvastatin therapy. However, serum cholesterol level was not significantly associated with patient survival. Furthermore, some meta-analyses were performed to integrate the findings from these studies on KTRs.

To our knowledge, only a few meta-analyses have been designed to investigate the effects of statins on KTRs, but the conclusions from these studies are controversial. Palmer et al. [5] argued that statins significantly reduced serum TC, LDL-C, HDL-C, and TG, but they had no effect on overall mortality, stroke, kidney function, and toxicity outcomes in KTRs. Rostami et al. [21] showed that the use of statins correlated independently with improved patient and graft survival after kidney transplantation. However, Messow and Isles [6] showed that it is not convincing that statins lower lipid in patients after renal transplant. Krista [22] argued that the use of statins cannot lower acute rejection risk after kidney transplantation. The evidence of use of statins in KTRs is insufficient. According to our meta-analysis, statins significantly lowered the serum level of TC and LDL-C but had no effect on serum HDL-C and TG in patients who underwent kidney transplantation. It is likely that the nature and mechanisms of dyslipidemia and the features of CVD in KTRs lead to different conclusions. This implies that further studies should focus on the mechanisms of lipid disorders and the effect of statin therapy in KTRs.

In renal transplant patients, lipid disorders play a major role in renal dysfunction. This is associated with increased plasma levels of TC, LDL-C, VLDL-C, and TG as well as decreased plasma levels of HDL-C. Many factors can influence posttransplant lipid profile including age, genetic predisposition, obesity, reduced physical activity, diet, and use of immunosuppressive agents (including calcineurin inhibitors, primarily cyclosporine, corticosteroids, and mammalian target of rapamycin inhibitors) [23]. Interventions targeting dyslipidemia are advocated because it has the most fatal adverse effects among the risk factors of CVD. CVD is the most common cause of death in renal transplant patients [24]. Moreover, death with a functioning graft (with CVD as the most common cause of death) is the overall most common cause of graft loss [25]. The proportion of the death with a functioning graft has increased over the 2 decades. And infections are the most leading causes that have not changed significantly [26]. In patients with end-stage renal disease, approximately $50 \%$ mortality is due to CVD and the incidence of CVD in KTRs is 4-6 times higher than in agematched individuals $[27,28]$. Lipoprotein (a) is a modified form of LDL which regulates inflammation of the endothelium and thrombosis and binds macrophages to promote foam cell formation leading to the deposition of cholesterol in atherosclerotic plaques. In this way, it aggravates tissue injury and increases the risk of atherosclerosis and CVDrelated morbidity and mortality in KTRs [29, 30]. Therefore, future investigations are needed to develop interventions that manage lipid abnormalities.

Several agents have been designed to regulate lipid profile such as statins, niacin, fibrates, ezetimibe, fish oil/omega-3, 


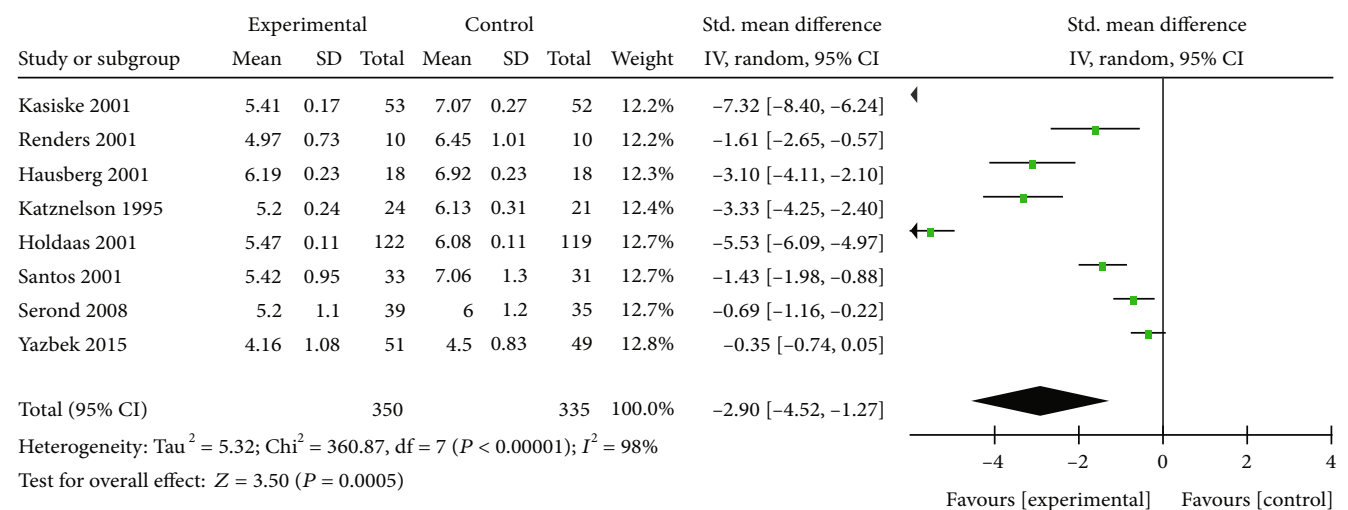

Figure 3: Forest plot of TC in KTRs.

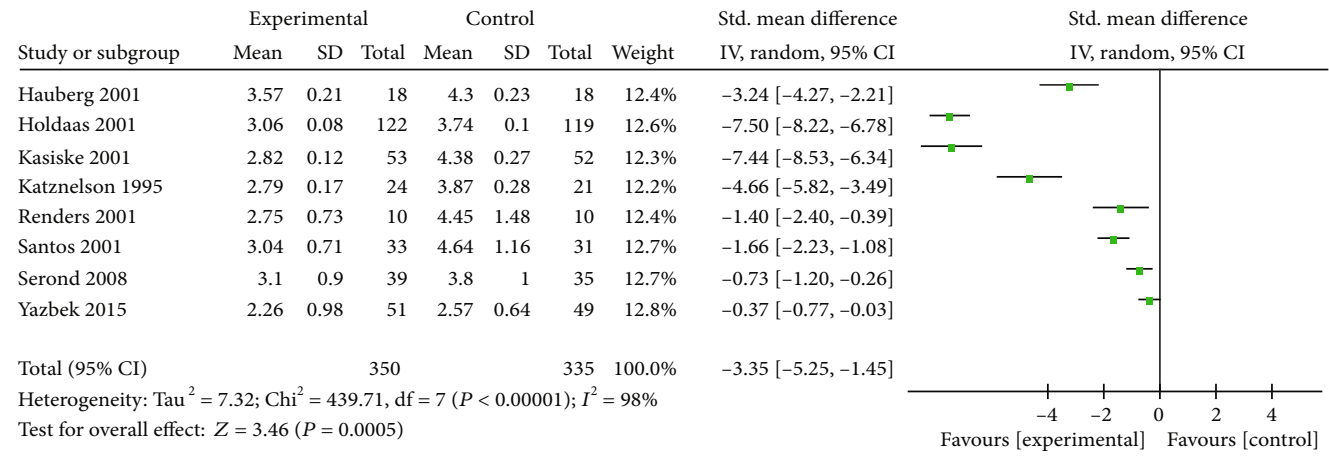

FIgURE 4: Forest plot of LDL-C in KTRs.

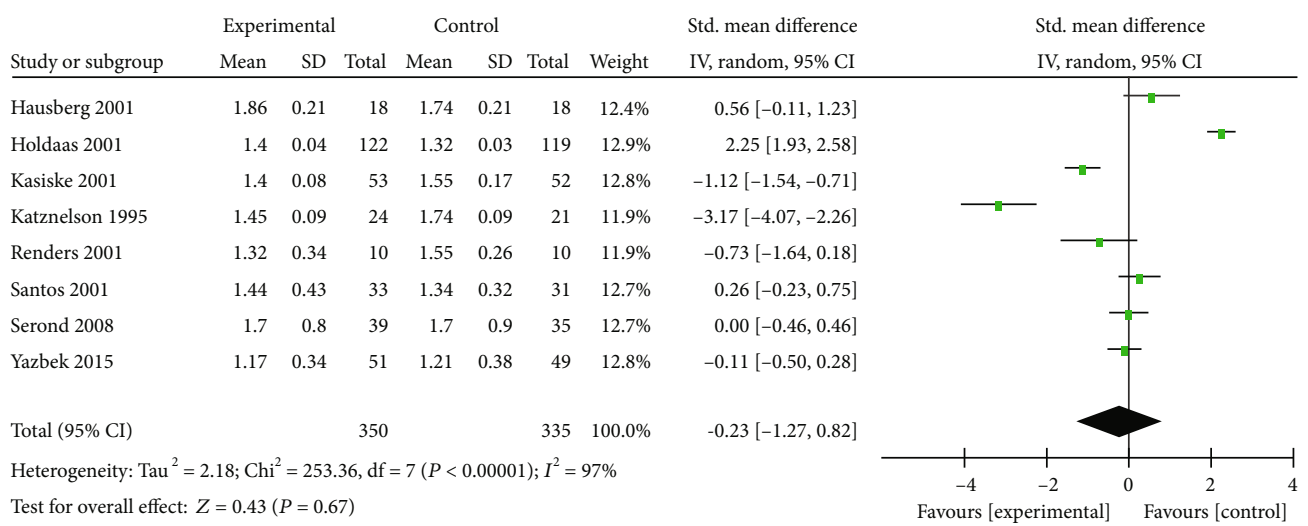

Figure 5: Forest plot of HDL-C in KTRs.

bile acid resins, and PCSK9 inhibitors [31-34]. So far, a number of studies have confirmed that statins are effective in lowering serum lipids and improving the cardiovascular risk compared to all other classes of medicines used in renal transplant patients [35]. Thus, both the kidney disease improving global outcomes (KDIGO) work group and European best practice guidelines for renal transplantation recommend the use of statins in adults who have received kidney transplant [36-38]. In the past few years, several studies have identified the benefits of statins on survival of patients and reducing the risk of cardiovascular complications in KTRs. For instance, a study [39] found that KTRs treated with statins have a $24 \%$ higher survival rate than patients who do not receive statins. Another study [40] reported that the use of statin therapy in patients treated with tacrolimus after kidney transplant significantly lowered the risk of major adverse cardiovascular events. Recently, Co et al. [41] also found that a statistically significant survival benefit was associated with the use of statins in a long-term follow-up retrospective study involving 687 ESRD patients 


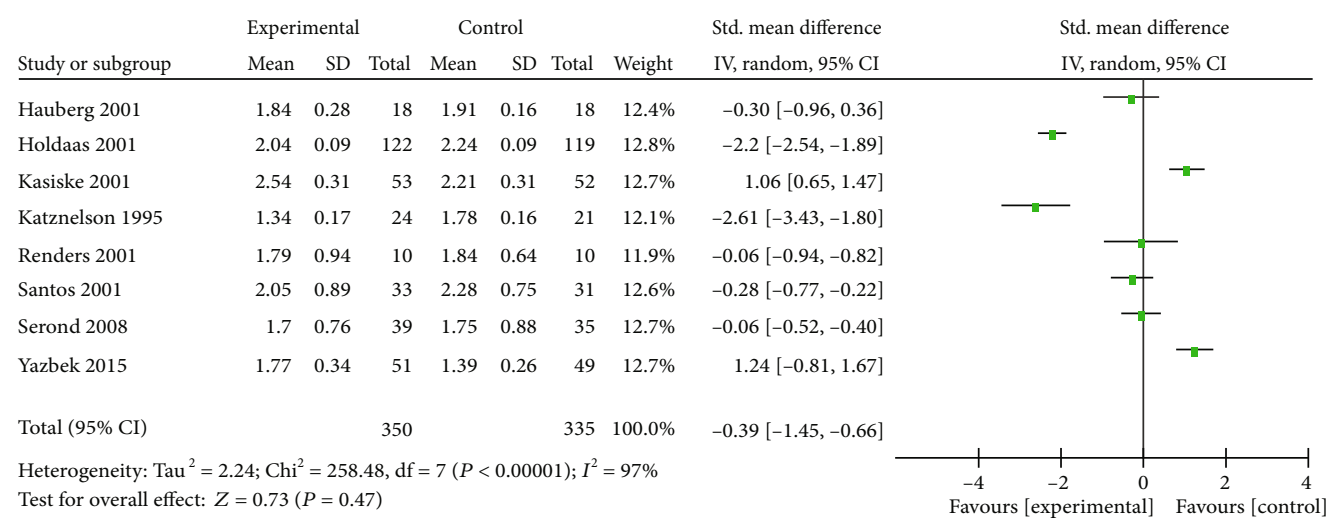

FIgURE 6: Forest plot of TG in KTRs.

who eventually underwent kidney transplant. Compelling evidence indicates that statins can be used to lower serum cholesterol (mainly aiming at LDL-C) after exercise and a low-fat diet has no effect on improving hypercholesterolemia. However, immunosuppressive agents are essential for KTRs. This is followed by adverse reactions, such as lipid metabolism disorders. Kidney transplantation and the use of immunosuppressive agents also contribute to lipid disorders [42]. For example, the use of chronic corticosteroid is associated with increases in TC, TG, and HDL-C [43]. Therefore, the effect of statins is more significant in KTRs. In addition to the cholesterol-lowering effects, statins also exert antiinflammatory and antiproliferative effects, plaque-stabilizing capacities, endothelial function improvement, antioxidant effects, and immunosuppressive effects and regulate monocyte recruitment, matrix deposition, and renal hemodynamics $[5,44-46]$. In addition, the anti-inflammatory effect of statins should not be ignored. Results from the studies demonstrate the ability of statins that improve graft outcome in the first year posttransplantation and inhibit transplant rejection $[47,48]$. Besides statins' established role in the management of lipid profile, it may be closely attributed to the various anti-inflammatory effects of statins. It was proved that statins can impinge on chemokine and inflammatory cytokine release, on prostaglandin expression, and on effector phagocytic function of monocytes [49, 50]. Moreover, statins also could ameliorate complement-mediated vascular damage that is vital to the initiation and perpetuation of inflammation [51]. Therefore, not only lipid-lowering but also anti-inflammatory effect is beneficial for the reduction of rejection episodes that are associated with enhancing graft longevity. As the rate-limiting step in cholesterol biosynthesis, HMG-CoA reductase is a major inhibition target through which statins reduce intracellular cholesterol in the liver, stimulate the expression of LDL receptors, and increase receptor-mediated endocytosis of $\mathrm{LDL}$, thereby lowering serum LDL and TC. It should be noted that there are other effects that mildly reduce TG and modestly elevate HDL. We should better take into consideration that dyslipidemia may persist a long time after renal transplantation, though statin treatment is persistent [52].So it is necessary to evaluate cholesterol levels regularly (including TC, LDL-C, HDL-C, and TG). As we all know, the methods for LDL-C measurement is progressing such as ultracentrifugation, the Friedewald equation, and the Martin-Hopkins equation $[53,54]$. Although these methods of LDL-C are alternative, the results are not very different. Moreover, we did choose the SMDs to eliminate the differences in some aspects.

Before prescribing statins to decrease lipid profile, secondary factors that elevate lipids such as nephrotic syndrome, hypothyroidism, diabetes mellitus, excessive alcohol intake, chronic liver disease, and other medication-induced dyslipidemias should be excluded [55].

4.2. Strengths and Limitations of the Study. This metaanalysis has some potential limitations. Firstly, some factors that influence heterogeneity such as race, gender, baseline serum lipid levels, use of different statins, different dosages and durations of statin therapy, duration of follow-up, and the nature of patient population were not analyzed. Secondly, only pooling eight studies were used to analyze the effects of statins on KTR subjects. Thirdly, it is regretful that we cannot get the eligible studies from the Chinese database.

\section{Conclusion}

Statin therapy significantly improves lipid profile (such as decreasing TG and LDL-C) in patients with KTRs. Therefore, statins should be prescribed to KTRs. However, more highquality, large-scale randomized controlled trials are needed to explore the effect of statin therapy in patients with dyslipidemia after renal transplantation. In addition, the underlying mechanisms responsible for dyslipidemia such as the type of immunosuppressant used require further investigation. Furthermore, the ongoing clinical trials should evaluate the adverse events of statins.

\section{Conflicts of Interest}

The authors declare that they have no conflicts of interest.

\section{Authors' Contributions}

Xiu Huang and Yong Jia should be considered joint first authors. 


\section{Acknowledgments}

We would like to thank Qiang Zhang for his valuable suggestions and statistical review.

\section{Supplementary Materials}

The following two results are, respectively, Embase and SinoMed search strategies. (Supplementary Materials)

\section{References}

[1] A. Lindholm, D. Albrechtsen, L. Frodin, G. Tufveson, N. H. Persson, and G. Lundgren, "Ischemic heart disease-major cause of death and graft loss after renal transplantation in Scandinavia," Transplantation, vol. 60, no. 5, pp. 451-457, 1995.

[2] C. Rigatto, "Clinical epidemiology of cardiac disease in renal transplant recipients," Seminars in Dialysis, vol. 16, no. 2, pp. 106-110, 2003.

[3] K. L. Lentine, S. P. Costa, M. R. Weir et al., "Cardiac disease evaluation and management among kidney and liver transplantation candidates: a scientific statement from the American Heart Association and the American College of Cardiology Foundation: endorsed by the American Society of Transplant Surgeons, American Society of Transplantation, and National Kidney Foundation," Circulation, vol. 126, no. 5, pp. 617-663, 2012.

[4] D. Del Castillo, J. M. Cruzado, J. M. Díaz et al., "The effects of hyperlipidaemia on graft and patient outcome in renal transplantation," Nephrology Dialysis Transplantation, vol. 19, no. 3, pp. iii67-iii71, 2004.

[5] S. C. Palmer, S. D. Navaneethan, J. C. Craig et al., "Hmg coa reductase inhibitors (statins) for kidney transplant recipients," The Cochrane Database of Systematic Reviews, no. article Cd005019, 2014.

[6] C. M. Messow and C. Isles, "Meta-analysis of statins in chronic kidney disease: who benefits?," QJM, vol. 110, no. 8, pp. 493500, 2017.

[7] W. G. Melsen, M. C. Bootsma, M. M. Rovers, and M. J. Bonten, "The effects of clinical and statistical heterogeneity on the predictive values of results from meta-analyses," Clinical Microbiology and Infection, vol. 20, no. 2, pp. 123-129, 2014.

[8] J. P. Ioannidis, N. A. Patsopoulos, and E. Evangelou, "Uncertainty in heterogeneity estimates in meta-analyses," $B M J$, vol. 335, no. 7626, pp. 914-916, 2007.

[9] M. Hausberg, M. Kosch, F. Stam et al., "Effect of fluvastatin on endothelium-dependent brachial artery vasodilation in patients after renal transplantation," Kidney International, vol. 59, no. 4, pp. 1473-1479, 2001.

[10] H. Holdaas, A. G. Jardine, D. C. Wheeler et al., "Effect of fluvastatin on acute renal allograft rejection: a randomized multicenter trial," Kidney International, vol. 60, no. 5, pp. 1990-1997, 2001.

[11] B. L. Kasiske, K. L. Heim-Duthoy, G. G. Singer, B. Watschinger, M. J. Germain, and B. Bastani, "The effects of lipid-lowering agents on acute renal allograft rejection," Transplantation, vol. 72, no. 2, pp. 223-227, 2001.

[12] S. Katznelson, A. H. Wilkinson, J. A. Kobashigawa et al., "The effect of pravastatin on acute rejection after kidney transplantation-a pilot study," Transplantation, vol. 61, no. 10, pp. 1469-1474, 1996.
[13] L. Renders, I. Mayer-Kadner, C. Koch et al., "Efficacy and drug interactions of the new HMG-CoA reductase inhibitors cerivastatin and atorvastatin in CsA-treated renal transplant recipients," Nephrology, Dialysis, Transplantation, vol. 16, no. 1, pp. 141-146, 2001.

[14] A. F. Santos, E. Keitel, A. E. Bittar et al., "Safety and efficacy of simvastatin for hyperlipidemia in renal transplant recipients: a double-blind, randomized, placebo-controlled study," Transplantation Proceedings, vol. 33, no. 1-2, pp. 1194-1195, 2001.

[15] D. Serón, F. Oppenheimer, L. M. Pallardó et al., "Fluvastatin in the prevention of renal transplant vasculopathy: results of a prospective, randomized, double-blind, placebo-controlled trial," Transplantation, vol. 86, no. 1, pp. 82-87, 2008.

[16] D. C. Yazbek, A. B. d. Carvalho, C. S. Barros, J. O. Medina Pestana, and M. E. F. Canziani, "Effect of statins on the progression of coronary calcification in kidney transplant recipients," PLoS One, vol. 11, no. 4, article e0151797, 2016.

[17] B. L. Kasiske, "Epidemiology of cardiovascular disease after renal transplantation," Transplantation, vol. 72, Supplement 6, pp. S5-S8, 2001.

[18] C. Wanner, V. Krane, W. März et al., "Atorvastatin in patients with type 2 diabetes mellitus undergoing hemodialysis," The New England Journal of Medicine, vol. 353, no. 3, pp. 238248, 2005.

[19] B. C. Fellström, A. G. Jardine, R. E. Schmieder et al., "Rosuvastatin and cardiovascular events in patients undergoing hemodialysis," The New England Journal of Medicine, vol. 360, no. 14, pp. 1395-1407, 2009.

[20] H. Holdaas, B. Fellström, A. G. Jardine et al., "Effect of fluvastatin on cardiac outcomes in renal transplant recipients: a multicentre, randomised, placebo-controlled trial," The Lancet, vol. 361, no. 9374, pp. 2024-2031, 2003.

[21] Z. Rostami, M. Moteshaker Arani, M. Salesi, M. Safiabadi, and B. Einollahi, "Effect of statins on patients and graft survival in kidney transplant recipients: a survival meta-analysis," Iranian Journal of Kidney Diseases, vol. 11, no. 5, pp. 329-338, 2017.

[22] K. L. Lentine and D. C. Brennan, "Statin use after renal transplantation: a systematic quality review of trial-based evidence," Nephrology Dialysis Transplantation, vol. 19, no. 9, pp. 23782386, 2004.

[23] I. Mikolasevic, M. Zutelija, V. Mavrinac, and L. Orlic, "Dyslipidemia in patients with chronic kidney disease: etiology and management," International Journal of Nephrology and Renovascular Disease, vol. 10, pp. 35-45, 2017.

[24] A. A. Awan, J. Niu, J. S. Pan et al., "Trends in the causes of death among kidney transplant recipients in the United States (1996-2014)," American Journal of Nephrology, vol. 48, no. 6, pp. $472-481,2018$.

[25] J. Noble, T. Jouve, P. Malvezzi, C. Süsal, and L. Rostaing, "Transplantation of marginal organs: immunological aspects and therapeutic perspectives in kidney transplantation," Frontiers in Immunology, vol. 10, p. 3142, 2020.

[26] J. Vega, C. Videla, H. Borja, H. Goecke, F. Martinez, and P. Betancour, "Causes of death with a functioning graft among kidney allograft recipients," Revista médica de Chile, vol. 140, no. 3, pp. 295-304, 2012.

[27] P. A. McCullough and W. C. Roberts, "Influence of chronic renal failure on cardiac structure," Journal of the American College of Cardiology, vol. 67, no. 10, pp. 1183-1185, 2016.

[28] W. Annema, A. Dikkers, J. Freark de Boer et al., "Hdl cholesterol efflux predicts graft failure in renal transplant recipients," 
Journal of the American Society of Nephrology, vol. 27, no. 2, pp. 595-603, 2016.

[29] N. Maharjan, U. Bedi, R. Arora, S. Bhandari, P. Dahal, and S. Khosla, "Impact of statins on cardiovascular outcomes in renal transplant recipients: a systematic review," American Journal of Therapeutics, vol. 18, no. 3, pp. e48-e54, 2011.

[30] H. Moradi, E. Streja, and N. D. Vaziri, "Esrd-induced dyslipidemia-should management of lipid disorders differ in dialysis patients?" Seminars in Dialysis, vol. 31, no. 4, pp. 398-405, 2018.

[31] L. Tannock, "Dyslipidemia in chronic kidney disease," in Endotext [Internet], K. R. Feingold, B. Anawalt, A. Boyce, G. Chrousos, K. Dungan, A. Grossman, J. M. Hershman, G. Kaltsas, C. Koch, P. Kopp, M. Korbonits, R. McLachlan, J. E. Morley, M. New, L. Perreault, J. Purnell, R. Rebar, F. Singer, D. L. Trence, A. Vinik, and D. P. Wilson, Eds., MDText.com, Inc., South Dartmouth (MA), 2000.

[32] N. Dincer, T. Dagel, B. Afsar, A. Covic, A. Ortiz, and M. Kanbay, "The effect of chronic kidney disease on lipid metabolism," International Urology and Nephrology, vol. 51, no. 2, pp. 265-277, 2019.

[33] B. Zheng-Lin and A. Ortiz, "Lipid management in chronic kidney disease: systematic review of PCSK9 targeting," Drugs, vol. 78, no. 2, pp. 215-229, 2018.

[34] A. K. H. Lim, K. J. Manley, M. A. Roberts, M. B. Fraenkel, and Cochrane Kidney and Transplant Group, "Fish oil for kidney transplant recipients," The Cochrane Database Of Systematic Reviews, no. article Cd005282, 2016.

[35] D. Leite and A. H. Campos, "A strategy to improve the cardiovascular risk factor profile in renal transplant patients," Arquivos Brasileiros de Cardiologia, vol. 94, no. 6, pp. 738-746, 2010.

[36] M. R. Hager, A. D. Narla, and L. R. Tannock, "Dyslipidemia in patients with chronic kidney disease," Reviews in Endocrine \& Metabolic Disorders, vol. 18, no. 1, pp. 29-40, 2017.

[37] C. Wanner, M. Tonelli, and the Kidney Disease: Improving Global Outcomes Lipid Guideline Development Work Group Members, "KDIGO clinical practice guideline for lipid management in CKD: summary of recommendation statements and clinical approach to the patient," Kidney International, vol. 85, no. 6, pp. 1303-1309, 2014.

[38] European best practice guidelines for renal transplantation, "Section iv: long-term management of the transplant recipient," Nephrology Dialysis Transplantation, vol. 17, Supplement 4, p. 3, 2002.

[39] F. G. Cosio, T. E. Pesavento, R. P. Pelletier et al., "Patient survival after renal transplantation iii: the effects of statins," American Journal of Kidney Diseases, vol. 40, no. 3, pp. 638643, 2002.

[40] N. Han, S. H. Han, Y. K. Song et al., "Statin therapy for preventing cardiovascular diseases in patients treated with tacrolimus after kidney transplantation," Therapeutics and Clinical Risk Management, vol. 13, pp. 1513-1520, 2017.

[41] M. L. F. Co, A. C. Agdamag, M. Z. Co, M. Hertl, and B. Mohamedali, "Intensity-dependent benefit of statins in survival among prospective kidney transplant patients," The American Journal of Cardiology, vol. 123, no. 2, pp. 254-259, 2019.

[42] S. Voora and D. B. Adey, "Management of kidney transplant recipients by general nephrologists: core curriculum 2019," American Journal of Kidney Diseases, vol. 73, no. 6, pp. 866879, 2019.
[43] G. A. Spinelli, C. R. Felipe, S. I. Park, E. L. Mandia-Sampaio, H. Tedesco-Silva Jr., and J. O. Medina-Pestana, "Lipid profile changes during the first year after kidney transplantation: risk factors and influence of the immunosuppressive drug regimen," Transplantation Proceedings, vol. 43, no. 10, pp. 3730 3737, 2011.

[44] M. Tonelli, C. Isles, G. C. Curhan et al., "Effect of pravastatin on cardiovascular events in people with chronic kidney disease," Circulation, vol. 110, no. 12, pp. 1557-1563, 2004.

[45] M. C. Ruiz, J. M. Moreno, N. Ruiz, F. Vargas, C. Asensio, and A. Osuna, "Effect of statin treatment on oxidative stress and renal function in renal transplantation," Transplantation Proceedings, vol. 38, no. 8, pp. 2431-2433, 2006.

[46] J. Rysz, A. Gluba-Brzozka, M. Banach, and A. Wiecek, "Should we use statins in all patients with chronic kidney disease without dialysis therapy? The current state of knowledge," International Urology and Nephrology, vol. 47, no. 5, pp. 805-813, 2015.

[47] R. Masterson, T. Hewitson, M. Leikis, R. Walker, S. Cohney, and G. Becker, "Impact of statin treatment on 1-year functional and histologic renal allograft outcome," Transplantation, vol. 80, no. 3, pp. 332-338, 2005.

[48] A. Shimabukuro-Vornhagen, J. Glossmann, T. Liebig, C. Scheid, and M. Bergwelt-Baildon, "The use of statins in hematopoietic stem cell transplantation," Current Stem Cell Research \& Therapy, vol. 4, no. 4, pp. 260-265, 2009.

[49] P. Ji, M. S. Si, Y. Podnos, H. Chow, E. Steward, and D. K. Imagawa, "Prevention of chronic rejection by pravastatin in a rat kidney transplant model," Transplantation, vol. 74, no. 6, pp. 821-827, 2002.

[50] D. W. McCarey, N. Sattar, and I. B. McInnes, "Do the pleiotropic effects of statins in the vasculature predict a role in inflammatory diseases?," Arthritis Research \& Therapy, vol. 7, no. 2, pp. 55-61, 2005.

[51] J. C. Mason, Z. Ahmed, R. Mankoff et al., "Statin-induced expression of decay-accelerating factor protects vascular endothelium against complement-mediated injury," Circulation Research, vol. 91, no. 8, pp. 696-703, 2002.

[52] P. Anagnostis, S. A. Paschou, E. Spartalis, G. Sarno, P. de Rosa, and G. Muscogiuri, "Metabolic complications and kidney transplantation: focus on glycaemia and dyslipidaemia," Current Vascular Pharmacology, vol. 17, 2019.

[53] W. T. Friedewald, R. I. Levy, and D. S. Fredrickson, "Estimation of the concentration of low-density lipoprotein cholesterol in plasma, without use of the preparative ultracentrifuge," Clinical Chemistry, vol. 18, no. 6, pp. 499-502, 1972.

[54] S. S. Martin, M. J. Blaha, M. B. Elshazly et al., "Comparison of a novel method vs the Friedewald equation for estimating lowdensity lipoprotein cholesterol levels from the standard lipid profile," Journal of the American Medical Association, vol. 310, no. 19, pp. 2061-2068, 2013.

[55] L. V. Riella, S. Gabardi, and A. Chandraker, "Dyslipidemia and its therapeutic challenges in renal transplantation," American Journal of Transplantation, vol. 12, no. 8, pp. 1975-1982, 2012. 